\title{
26-Gb/s DMT Transmission Using Full C-Band Tunable VCSEL for Converged PONs
}

\section{Wagner, Christoph; Dochhan, Annika ; Eiselt, Michael H.; Grobe, Klaus; Ortsiefer, Markus; Greus, Christoph; Neumeyr, Christian; Paul, Sujoy; Cesar, Julijan; Küppers, Franko}

Total number of authors:

12

Published in:

I E E E Photonics Technology Letters

Link to article, DOI:

10.1109/LPT.2017.2710323

Publication date:

2017

Document Version

Peer reviewed version

Link back to DTU Orbit

Citation (APA):

Wagner, C., Dochhan, A., Eiselt, M. H., Grobe, K., Ortsiefer, M., Greus, C., Neumeyr, C., Paul, S., Cesar, J., Küppers, F., Vegas Olmos, J. J., \& Tafur Monroy, I. (2017). 26-Gb/s DMT Transmission Ussing Full C-Band Tunable VCSEL for Converged PONs. I E E E Photonics Technology Letters, 29(17), 1475-1478. https://doi.org/10.1109/LPT.2017.2710323

\section{General rights}

Copyright and moral rights for the publications made accessible in the public portal are retained by the authors and/or other copyright owners and it is a condition of accessing publications that users recognise and abide by the legal requirements associated with these rights.

- Users may download and print one copy of any publication from the public portal for the purpose of private study or research.

- You may not further distribute the material or use it for any profit-making activity or commercial gain

- You may freely distribute the URL identifying the publication in the public portal 


\title{
26-Gbps DMT Transmission using Full C-band Tunable VCSEL for Converged PONs
}

\author{
Christoph Wagner, Annika Dochhan, Member, IEEE, Michael H. Eiselt, Fellow, OSA, Senior \\ Member, IEEE, Klaus Grobe, Senior Member, IEEE, Markus Ortsiefer, Christoph Gréus, Christian \\ Neumeyr, Sujoy Paul, Julijan Cesar, Franko Küppers, Juan José Vegas Olmos, Senior Member, IEEE, \\ and Idelfonso Tafur Monroy, Senior Member, IEEE
}

\begin{abstract}
WDM-PON is considered for converged fixed mobile broadband access networking. We propose to utilize lowcost tunable lasers at the remote sites, together with a centralized wavelength locker. Practical implementations require a transparently added downstream signaling channel and upstream per-channel pilot tones for channel tagging and remote wavelength control. We demonstrate, for the first time, $26 \mathrm{Gbps}$ discrete multitone transmission modulated on a low-cost wide tunable vertical surface emitting laser over up to $40 \mathrm{~km}$ of standard single-mode fiber. The results confirm that converged fixed mobile WDM-PON systems based on low-cost lasers carrying discrete multitone modulation are a technically viable approach.
\end{abstract}

Index Terms - Discrete Multitone, WDM-PON, Tunable Laser

\section{INTRODUCTION}

$\mathrm{M}$ OBILE bandwidths are increasing by $53 \%$ per year in Western Europe [1]. Mobile network operators are consolidating and centralizing network functions to cope with the increased bandwidth demands on the air interface. This has led to a separation of radio frequency (RF) signal generation and processing in centralized baseband units (BBUs) and RFband / baseband conversion in remote radio heads (RRHs). However, this split imposes stringent bandwidth and latency

Manuscript received January XX, 2017. This work was supported in part by the Marie Curie Initial Training Network project ABACUS, the European Community's Seventh Framework Program (FP7/2007-2013) under Grant 317762 (ICT-COMBO) and Horizon 2020 under Grant 671551 (5G-Xhaul), German ministry of education and research (BMBF) project VCSEL-Trx.

C. Wagner is with the Technical University of Denmark, Department of Photonics Engineering, 2800 Kgs. Lyngby, Denmark, and ADVA Optical Networking SE, 98617, Meiningen, Germany (e-mail: chwag@ fotonik.dtu.dk).

M. H. Eiselt, and A. Dochhan are with ADVA Optical Networking SE, $98617 \quad$ Meiningen, Germany (meiselt@advaoptical.com; adochhan@advaoptical.com).

K. Grobe is with ADVA Optical Networking SE, 82152 Martinsried, Germany. (e-mail: kgrobe@ advaoptical.com).

Markus Ortsiefer, Christoph Greus, and Christian Neumeyr are with Vertilas GmbH, Daimlerstrasse 11d, 85748 Garching, Germany (e-mail: ortsiefer@vertilas.com; greus@ vertilas.com; neumeyr@vertilas.com)

Sujoy Paul, Julijan Cesar, and Franko Küppers, are with Technical University Darmstadt, Institute for Microwave Engineering and Photonics, Merckstr. 25, 64283 Darmstadt, Germany (e-mail:paul@imp.tu-darmstadt.de; cesar@imp.tu-darmstadt.de; kueppers@imp.tu-darmstadt.de)

J. J. Vegas Olmos and I. Tafur Monroy are with DTU Fotonik, $2800 \mathrm{Kgs}$. Lyngby, Denmark. (e-mail: jjvo@fotonik.dtu.dk; idtm@fotonik.dtu.dk). requirements on the mobile fronthaul (MFH) system, which is the transport link between BBUs and RRHs, where protocols like the common public radio interface (CPRI) are used [2]. In addition, the integration of backhaul and fronthaul is ongoing. While mobile backhaul is based on Ethernet, nextgeneration radio access networks must be compatible with any of the related protocols. This is facilitated by fixed mobile convergence (FMC).

A cost-efficient solution for FMC is the wavelength division multiplex (WDM) passive optical network (PON). The standardization group ITU-T SG15/Q6 is currently defining a unified network serving MFH, mobile backhaul and enterprise access in the recommendation draft G.metro. One of the key features of this WDM-PON system is a centralized wavelength controller in the optical line terminal (OLT) enabling remote wavelength tuning of the tunable laser in the optical network unit (ONU) [3].

Due to the high bandwidth growth in the mobile network, soon CPRI option 10 (24.33 Gbps) will be necessary to be deployed. To keep lowest costs for FMC access networks, higher order intensity modulation formats with direct detection, like discrete multitone (DMT) or pulse amplitude modulation with four levels (PAM-4), are necessary to support high data rates transmitted via low bandwidth equipment.

In this letter, we present for the first time, a CPRI option 10 capable transmission based on a wideband tunable MEMSVCSEL (micro-electro-mechanical system vertical cavity surface emitting laser) over $40 \mathrm{~km}$ using DMT. Details of the packaged MEMS-VCSEL are also presented. Results show that DMT modulated on MEMS-VCSEL based WDM-PON is a viable solution to cope with the pressing FMC needs.

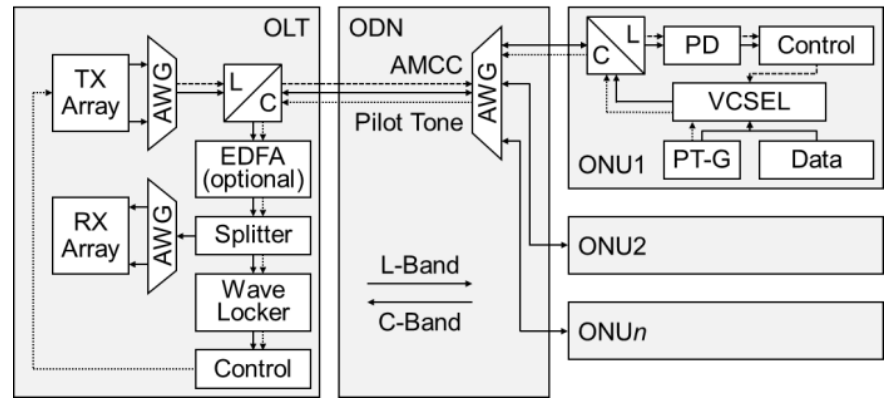

Fig. 1. WDM-PON system based on low-cost tunable lasers. TX: transmitter, RX: receiver, AWG: arrayed waveguide grating, PD: photodetector, PT-G: pilot-tone generator, VCSEL: vertical cavity surface emitting laser. 


\section{TUNABLE WDM-PON SYSTEM CONCEPT}

For FMC WDM-PON systems, preferably low-cost tunable lasers are used in the ONU. For cost reduction, these lasers should be not calibrated and have no integrated wave locker. A possible G.metro system scheme is shown in Fig. 1. This system uses pilot tones (PTs) as channel label and an auxiliary management communication channel (AMCC) as wavelength control feedback loop. Details are discussed in [4].

In NG-PON2, an AMCC is also implemented in the upstream direction to remotely monitor and manage the ONU. In G.metro, this approach is also intended to be used. Currently, AMCC bit rates of $50 \mathrm{kbps}$ and pilot tones around $50 \mathrm{kHz}$ are under discussion.

DMT, as payload data modulation scheme, is based on multiple subcarriers. The subcarrier frequency distance $\Delta f_{\text {Sub }}$ is determined by the (inverse) fast Fourier transformation ((i)FFT) length $N$ and sampling frequency $f_{S}$ of the digital-toanalog-converter (DAC) by $\Delta f_{\text {sub }}=f_{S} / N .26$ Gbps transmission $(80 \mathrm{GS} / \mathrm{s})$ and an (i)FFT length of 512 points result in a first subcarrier frequency approximately at $150 \mathrm{MHz}$. The considered PTs frequency is 3000 times lower than the first DMT subcarrier. Therefore, only a very small penalty is expected due to the huge guard band. However, this applies only if the PT or AMCC is directly modulated onto the laser bias current leading to an added frequency component in the spectrum. For external modulation, e.g., by a Mach-Zehnder modulator, the PT or AMCC spectrum would be convolved with each DMT subcarrier, leading to higher expected penalties [4].

\section{10 GBPS WIDELY TUNABLE VCSEL}

The presented transmission experiment is based on a single mode widely tunable $1.55 \mu \mathrm{m}$ vertical cavity surface emitting laser diode (VCSEL) packaged in a transmit-opticalsubassembly (TOSA). The TOSA can be easily assembled inside standardized small form factor pluggable (SFP) optical modules. The tunable VCSEL is based on a long wavelength Indium Phosphide Buried Tunnel Junction VCSEL and features a MEMS top mirror pioneered by the Technical University of Darmstadt. A cross section of this VCSEL design is illustrated in Fig. 2. Details about the device structure and manufacturing can be found in. [5], [6]

The small air gap between the surface of the base VCSEL and the MEMS can be controlled via thermo-electrical effects. The change in air gap leads to mode-hop-free tuning of the

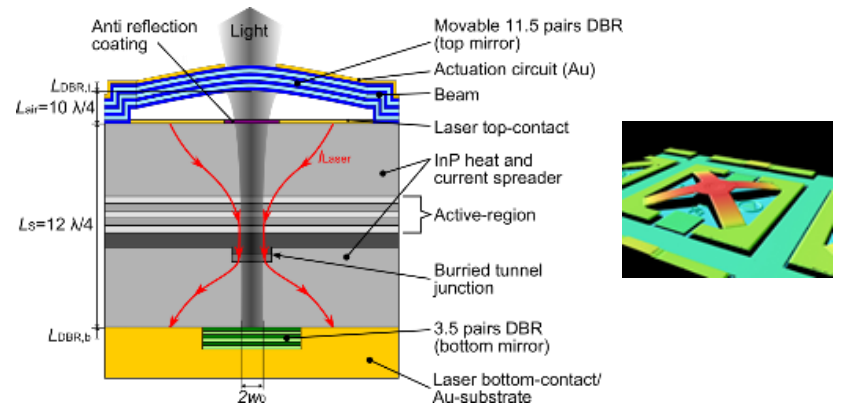

Fig. 3. MEMS-VCSEL cross section (left) and MEMS top view (right).

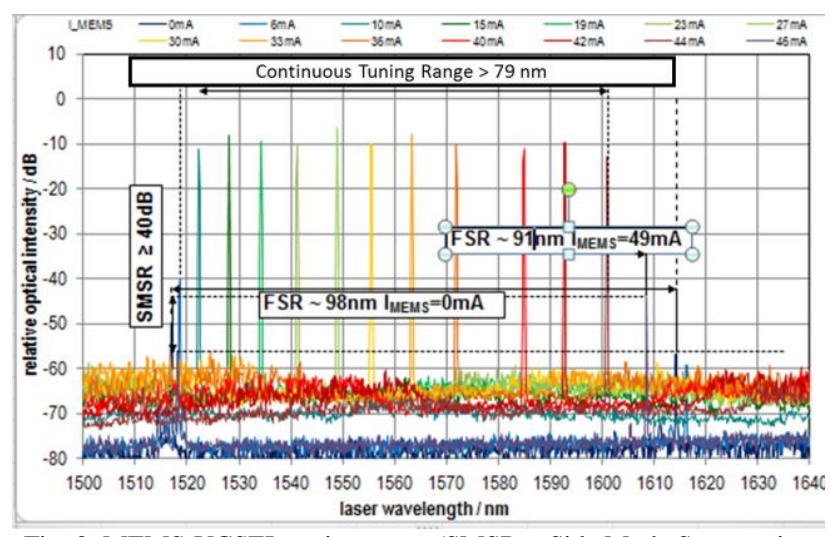

Fig. 2. MEMS-VCSEL tuning range $($ SMSR $=$ Side Mode Suppression Ratio, FSR $=$ Free Spectral Range) .

laser wavelength. The tunable VCSEL deployed for this experiment has a maximum tuning range from $1517 \mathrm{~nm}$ to $1608 \mathrm{~nm}$ (Fig. 3) and a peak optical power of $1.2 \mathrm{~mW}$ (fiber coupled).

For the transmission tests, the tuning range has been limited from $1530.33 \mathrm{~nm}$ to $1561.41 \mathrm{~nm}$. These wavelengths are a subset of the C-band specified in [7] and are the first and last channel of the used cyclic 40-channel $100-\mathrm{GHz}$ channel grid multiplexer (MUX) and demultiplexer (DEMUX). However, at $1530.33 \mathrm{~nm}$ emission wavelength a reduced reflectivity of the distributed Bragg reflector (DBR) leads to an increased threshold current. In the future, the design will be optimized to enable the use for both C- and L-band applications with one laser.

For the assembly of the tunable laser inside a TOSA, several components and functions needed to be integrated. Besides the tunable laser, the submount features $10 \mathrm{Gbps}$ capable contact pads, control pins to adjust the laser wavelength, and a thermistor to probe the laser temperature. Due to the tuning mechanism, only one control signal is required to tune the laser without mode hops across the full tuning range.

\section{UPSTREAm MEASUREMENT SETUP AND RESUltS}

Figure 4 shows the experimental setup. The electrical DMT signal is generated by using offline digital signal processing via Python routines. The used DMT parameters are summarized in Table I. DMT requires a real valued baseband signal. Hence, an FFT-length of 512 points leads to 255 usable subcarriers at maximum. Therefore, the first subcarrier frequency is at $156.86 \mathrm{MHz}$ leaving enough margin for adding a low frequency pilot tone as proposed in the system concept. To optimize the transmission performance for each subcarrier, bit and power loading is implemented using the LevinCampello algorithm [8]. For this purpose, in a first step, the signal-to-noise ratio (SNR) is estimated by using a 16-QAM constellation with equal power distribution. For high estimated SNR values, a higher-order modulation constellation is loaded onto the respective subcarrier (i.e., up to 16-QAM), BPSK for low estimated SNRs, or even no data are modulated onto the subcarrier if the SNR is below a certain threshold. Additionally, the power of each subcarrier is adjusted to 


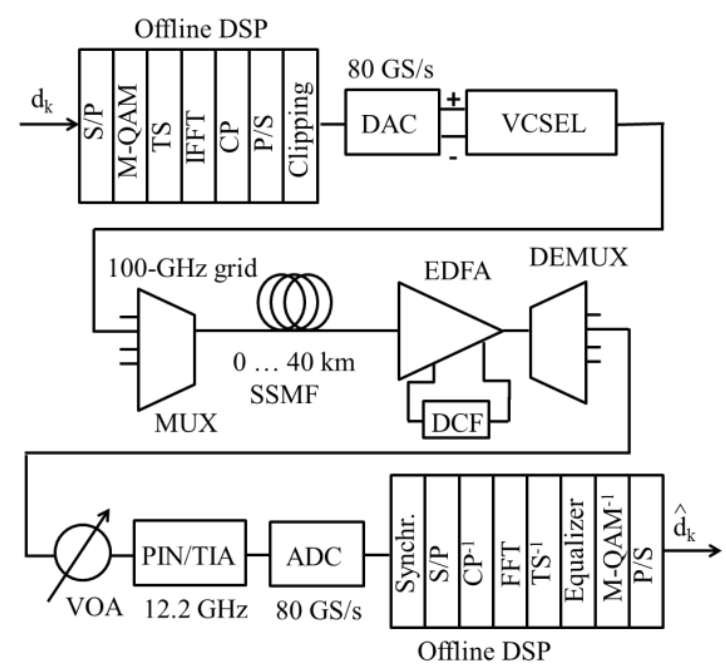

Fig. 4.Experimental setup for 26Gbps DMT transmission up to $40 \mathrm{~km} \mathrm{SSMF}$ transmission

achieve a homogeneous bit-error ratio (BER) for all used subcarriers. Five training symbols (TS) are added for synchronization and channel estimation. Finally, a 32 sample cyclic prefix (CP) is added and the signal is clipped symmetrically by $9 \mathrm{~dB}$ clipping ratio $c l_{\mathrm{dB}}$ to reduce the peakto-average power ratio. The clipped signal $x_{\mathrm{cl}}(t)$ defined by:

$$
x_{\mathrm{cl}}(t)=\left\{\begin{array}{ccc}
-10^{\frac{c l_{\mathrm{dB}}}{20}} \cdot \sigma_{x} & \text { if } & x(t)<-10^{\frac{c l_{\mathrm{dB}}}{20}} \cdot \sigma_{x} \\
x(t) & \text { if } & -10^{\frac{c l_{d B}}{20}} \cdot \sigma_{x} \leq x(t) \leq 10^{\frac{c l_{\mathrm{dB}}}{20}} \cdot \sigma_{x} \\
10^{\frac{c l_{\mathrm{dB}}}{20} \cdot} \cdot \sigma_{x} & \text { if } & x(t)>10^{\frac{c l_{\mathrm{dB}}}{20}} \cdot \sigma_{x}
\end{array}\right.
$$

With the unclipped DMT time signal $x(t)$ and the standard deviation $\sigma_{x}$ and was experimentally optimized.

The data rate is fixed to the CPRI option $10(24.33 \mathrm{Gbps})$ with $7 \%$ forward error correction (FEC) overhead resulting in 26 Gbps gross transmission rate. The shown DMT transmission rates are without TS and CP.

The electrical DMT signal is directly modulated onto the MEMS-VCSEL bias current via a high-speed DAC working at a sampling rate of $80 \mathrm{GS} / \mathrm{s}$. The differential output swing of the DAC is limited to $800 \mathrm{mV}$. The VCSEL's bandwidth is approximately $7 \mathrm{GHz}$ leading to bandwidth limitations, and its bias current is optimized for each measured wavelength between 17-19 mA. The high bandwidth DAC was available and used to make sure that no bandwidth limitations are introduced by this device. The optical wavelength is thermoelectrically tuned between $1530.33 \mathrm{~nm}$ and $1561.61 \mathrm{~nm}$. The optically modulated DMT signal is sent to a 40-channel MUX with $100 \mathrm{GHz}$ channel spacing, and each channel has an optical bandwidth of $78 \mathrm{GHz}$ leading to no optical bandwidth limitations. The optical signal is launched into the standard single mode fiber (SSMF) at $-8 \mathrm{dBm}$. After transmission over up to $40 \mathrm{~km}$ SSMF the signal is amplified by an ErbiumTABLE I

DMT PARAMETER OVERVIEW

\begin{tabular}{cc}
\hline \hline Parameter & Value \\
\hline Cyclic prefix & 32 samples \\
Clipping ratio & $9 \mathrm{~dB}$ \\
FFT length & 512 \\
TS & 5 \\
BL/PL & Levin-Campello \\
Usable carriers & 255 \\
\hline \hline
\end{tabular}

doped-fiber-amplifier (EDFA), and the dispersion is compensated with the same dispersion compensating fiber (DCF) for $50 \mathrm{~km}$ for all measured reaches. The MEMSVCSEL is strongly chirped which results in a poor tolerance against positive chromatic dispersion (CD). The combination of a fix overcompensating DCF in the OLT side and the strong chirp of the VCSEL enables a wide range of the transmission distances. The optical input power for the DCF is kept constant at $-4 \mathrm{dBm}$ to avoid nonlinearities in the DCF. Since DCF and EDFA can be shared between all channels of a WDM-PON system, this approach still supports the low cost requirements for FMC networks. The optical signal is sent through a DEMUX with similar properties as the MUX. A variable optical attenuator (VOA) is used to measure the BER over received power input into a linear PIN photodiode with integrated transimpedance amplifier (TIA) with $12.2 \mathrm{GHz}$ bandwidth, leading to further constraints in the electro-optical bandwidth. The opto-electrical converted signal is sent to an analog-to-digital converter with $80 \mathrm{GS} / \mathrm{s}$ which is connected to the offline DSP, where the data are recovered and the BER is measured after synchronization and removal of the CP and TS. For all wavelengths and transmission reaches, a low latency interleaved Reed-Salomon Bose-Chaudhuri-Hocquenghem enhanced FEC is assumed. For CPRI's target BER of $10^{-12}$ an input BER of $2.26 \cdot 10^{-3}$ is required [9].

\section{RESUltS AND DisCUSSION}

For the WDM-PON scheme with centralized wavelength locking, a widely tunable laser is required. We tested different wavelengths, i.e., the first, last, and one channel in the middle of the transmission band. The estimated SNR and bit loading for $0 \mathrm{~km}$ and $40 \mathrm{~km}$ SSMF transmission are shown in Fig. 5 . The usable bandwidth of the VCSEL can be seen from the estimated SNR and bit loading after $0 \mathrm{~km}$ transmission shown in Fig. 5a and 5c. The bandwidth was very similar for $1561.42 \mathrm{~nm}$ and $1545.32 \mathrm{~nm}$. At $1530.33 \mathrm{~nm}$ the estimated SNR is slightly reduced. The bit loading for $1530.33 \mathrm{~nm}$ after $0 \mathrm{~km}$ transmission is shown in Fig. 5c. The first subcarrier is not used due to AC coupling effects at both transmitter and receiver side. The usable bandwidth is approximately $10 \mathrm{GHz}$. Fig. $5 \mathrm{~b}$ and $5 \mathrm{~d}$ show the combined transmission effects and
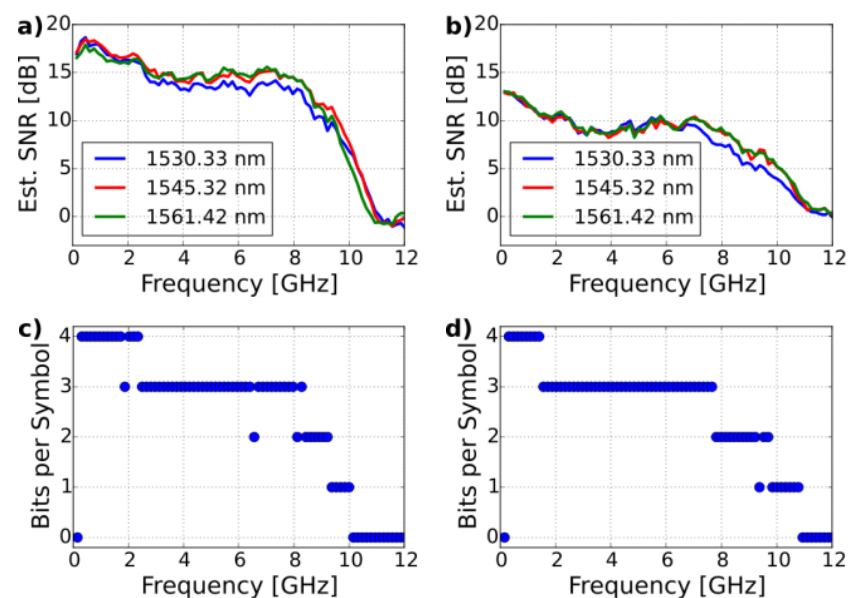

Fig. 5. a) Estimated SNR after $0 \mathrm{~km} \mathrm{SSMF}$ for 3 measured wavelengths and b) Estimated SNR after $40 \mathrm{~km}$ SSMF for 3 measured wavelengths. c) Bit loading for $1530 \mathrm{~nm}$ wavelength after $0 \mathrm{~km} \mathrm{SSMF}$. d) Bit loading for 1530 $\mathrm{nm}$ wavelength after $40 \mathrm{~km}$ SSMF. 


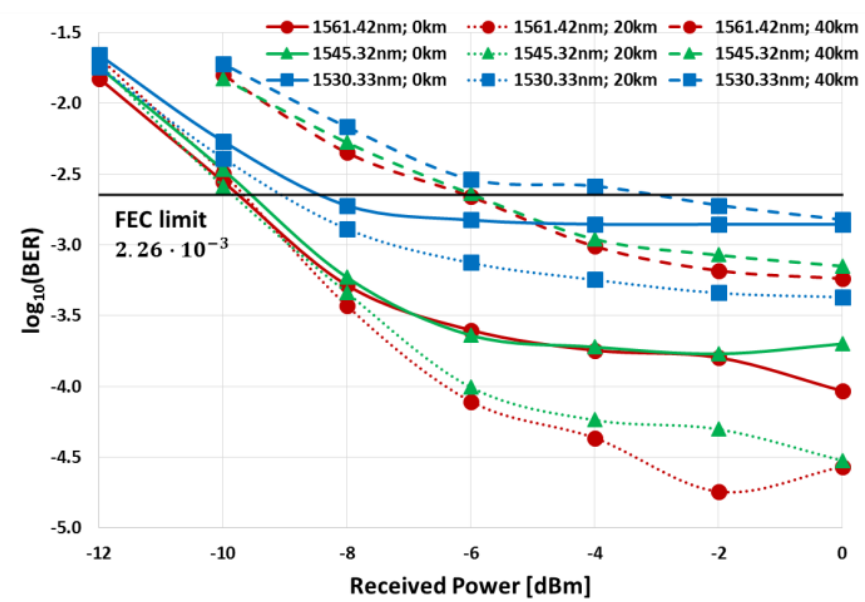

Fig. 6. BER vs. received power at $26 \mathrm{Gbps}$ for different wavelengths and SSMF transmission reaches.

bandwidth limitation after $40 \mathrm{~km}$ transmission. Although the estimated SNR is reduced, the bit loading schemes for $0 \mathrm{~km}$ and $40 \mathrm{~km}$ are very similar. A dispersion notch cannot be seen due to the bandwidth limitation of the VCSEL.

We also tested the most typical FMC transmission reaches. $20 \mathrm{~km}$ is the upper boundary for mobile fronthaul CPRI transmission, while $40 \mathrm{~km}$ is a typical reach for fixed enterprise access. The transmission results are shown in Fig. 6.

The FEC limit is attained for all tested wavelengths and transmission reaches. Interesting is the wavelength dependency. The performance at $1561.41 \mathrm{~nm}$ and $1545.32 \mathrm{~nm}$ is very similar for all tested transmission reaches. The similar error floors show that the laser chirp, laser nonlinearities, and residual dispersion are comparable for these wavelengths. The lower error floor at $20 \mathrm{~km}$ reach shows that the residual CD and the laser chirp optimally compensate for each other at this point. The slightly increased error floor in back-to-back transmission compared to the strongly increased error floor at $40 \mathrm{~km}$ transmission shows that the system is more robust against negative $\mathrm{CD}$.

In contrast to these two wavelengths, the shortest wavelength at $1530.33 \mathrm{~nm}$ shows an increased error floor. As described above, this wavelength is at the edge of the DBR mirror reflectivity leading to an increased laser threshold current and a reduced SNR. Furthermore, the linear region of the laser is reduced. For DMT, a linear transmitter is necessary to avoid subcarrier intermodulation products.

However, with the next generation of the MEMS-VCSEL we expect that the wavelength dependency over the whole $\mathrm{C}$ band is removed. In the meantime, half of the C-band can be used for the upstream and the other half for downstream. This reduces the wavelength dependency and helps to improve the reliability of the system.

Figure 7 shows the performance versus the transmission data rate. Neglecting the $1530.33 \mathrm{~nm}$ wavelength, we demonstrate that the system is able to transmit bit rates beyond $35 \mathrm{Gbps}$ on the longer wavelengths over $20 \mathrm{~km}$ transmission reach. This is beyond CPRI option 10. Taking into account that CPRI is currently looking into other physical-layer splits to reduce the necessary bitrate makes the proposed G.metro system with DMT modulation future-proof.

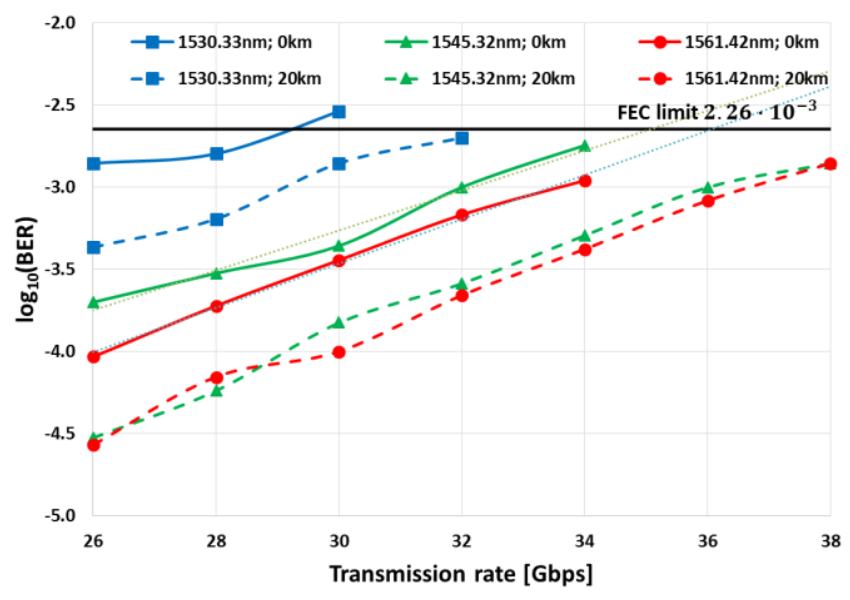

Fig. 7. BER vs. transmission data rate for different wavelengths up to $20 \mathrm{~km}$ SSMF transmission reach.

\section{CONCLUSIONS}

This letter provides an experimental demonstration of a lowcost WDM-PON system based on a DMT modulated MEMSVCSELs which overcomes the capacity crunch in the so called mobile fronthaul. We demonstrate, for the first time, $26 \mathrm{Gbps}$ DMT transmission over $40 \mathrm{~km}$ SSMF using a VCSEL with a tuning range of more than $30 \mathrm{~nm}$. Additive implementation of the pilot tone could lead to impairments for amplitude modulation schemes. Discrete multitone modulation is based on multiple subcarriers leading to little expected impairments when the pilot tone and the second subcarrier have a large frequency deviation. This is suitable for the proposed converged fixed and mobile network applications utilizing the G.metro novel WDM-PON system and extends the system application to converged mobile and enterprise access with differential reaches of up to $40 \mathrm{~km}$. For mobile networks of up to $20 \mathrm{~km}$, we demonstrate future proofed transmission beyond 35 Gbps.

\section{REFERENCES}

[1] T. Cisco, "Cisco Visual Networking Index : Global Mobile Data Traffic Forecast Update , 2010 - 2015," Growth Lakel., vol. 2011, no. 4, pp. 2010-2015, 2011.

[2] Public Radio Interface, "Common Public Radio Interface (CPRI); Interface Specification," 2013.

[3] K. Grobe, M. H. Eiselt, S. Pachnicke, and J.-P. Elbers, "Access Networks Based on Tunable Lasers," J. Light. Technol., vol. 32, no. 16, 2014, pp. 2815-2823.

[4] C. Wagner, M. H. Eiselt, M. Lawin, S. Zou, K. Grobe, J. J. Vegas Olmos, and I. Tafur Monroy, "Impairment Analysis of WDM-PON based on Low-Cost Tunable Lasers," J. Light. Technol., vol. 34, no. 22, 2016, pp. 5300-5307.

[5] S. Paul, J. Cesar, C.n Gierl, M. Tanvir Haidar, B. Koegel, C. Neumeyr, M. Ortsiefer, and F. Kueppers, "Ultra wide mode-hop free tuning around $1550 \mathrm{~nm}$ telecom wavelength using high-speed MEMS-VCSELs," J3, 20th Microoptics Conference, Fukuoka (2015).

[6] S. Paul, C. Gierl, J.Cesar, Q. T. Le, M. Malekizandi, F. Kueppers, B. Koegel, J. Rosskopf, C. Greus, M. Görblich, Y. Xu, C. Neumeyr, M. Ortsiefer, "High speed surface micromachined MEMS tunable VCSEL for telecom wavelengths," CLEO 2015

[7] ITU-T Recommendation G.698.3, "Multichannel seeded DWDM applications with single-channel optical interfaces" 2012.

[8] J. M. Cioffi, "Data Transmission Theory," course text for EE379C (http://www.stanford.edu/group/cioffi/).

[9] ITU-T Recommendation G.975.1, "Forward error correction for high bit-rate DWDM submarine systems - Appendix I.4," 2004. 